\title{
Primary PTCA in the UK: if not for all, then for whom?
}

\author{
Nicholas Brooks
}

Primary angioplasty (PTCA) is effective treatment for acute myocardial infarction, and randomised trials have suggested that a lower mortality and incidence of recurrent ischaemia and stroke can be achieved compared with thrombolysis.

However, the number of patients randomised into primary angioplasty trials is small and the magnitude of the advantage over thrombolysis correspondingly uncertain. The trials were conducted in centres with an exceptional commitment to angioplasty and did not employ the best thrombolytic regimen; accelerated tissue plasminogen activator ( $t-P A)$. The preliminary results of the larger GUSTO II trial, which were presented to the American College of Cardiology in April 1996, showed an advantage of angioplasty over accelerated t-PA at 30 days of one death, one cerebral haemorrhage, and two reinfarctions prevented for every hundred patients, which falls short of an unequivocal endorsement for angioplasty. ${ }^{1}$ As many as seven lives per hundred might be saved over 30 months by a reduction of one hour in the delay to thrombolysis. ${ }^{2}$ Finally, research into new adjunctive antithrombin therapies offers the promise of improved results from thrombolysis without necessarily incurring an increased risk of cerebral haemorrhage.

The daunting logistic implications of primary angioplasty are not confined to cost; training would have to be totally restructured if interventional expertise were to become a requirement for all cardiologists, and unpredictable interruption of clinics, ward rounds, and catheter laboratory sessions could be highly disruptive.

Primary angioplasty could be justified as routine treatment in centres with sufficient capacity to avoid such disruption of their elective workload, but the commitment required to match published results should not be underestimated. Delay adds greatly to risk and when the admission to balloon time is likely to exceed 120 minutes treatment should be with thrombolysis. ${ }^{3}$ Priority might be given to patients thought to have most to gain from primary angioplasty: those with cardiogenic shock or with a contraindication to thrombolysis, and those over the age of 65 who experienced the main benefit from angioplasty in the PAMI 2 trial. ${ }^{4}$

The only prospect for improved survival in cardiogenic shock is with early reperfusion. Thrombolytic therapy has proved to be ineffective. Several primary angioplasty series have reported higher survival rates than would have been anticipated with conventional management, and the recovery of cardiac function is sometimes dramatic. While favourable results may be over represented in the literature and patients treated by angioplasty have tended to be younger than those managed conservatively implying a bias in selection of lower risk patients for angioplasty, ${ }^{5}$ currently available evidence supports the use of emergency angioplasty for this lethal condition.

Mortality in patients considered ineligible for thrombolysis is up to five times higher than among those who receive treatment. Such patients tend to be older and to have other indications of being at high risk. ${ }^{6}$ A mortality of $24 \%$ has been reported even with angioplasty ${ }^{7}$ so that the benefit from intervention is far less certain than the overall mortality figures suggest. Nevertheless, the logic for offering effective treatment to patients in whom thrombolysis is contraindicated because of bleeding risk, in whom a mortality of $29 \%$ has been reported, ${ }^{6}$ and who would otherwise be untreated, seems unassailable. The case for primary angioplasty for other high risk patients such as those with diabetes, those over 75, and those whose electrocardiogram shows ST depression is persuasive but currently no good evidence exists to support the policy.

\section{Conclusions}

The available evidence is insufficient to argue for the massive restructuring of cardiac services that would be required to make primary angioplasty available to all patients with acute myocardial infarction. Even with more conclusive evidence the universal provision of angioplasty is an unrealistic goal. Strategies to enable rapid transfer to interventional centres of relatively small numbers of highly selected patients should, however, be explored and opportunistic angioplasty for patients admitted direct to interventional centres is justified.

1 Verheught FWA. Primary angioplasty for acute myocardial infarction: is the balloon half full or half empty? Lancet 1996;347:1276-7.

2 Rawles J. Magnitude of benefit from earlier thrombolytic treatment in acute myocardial infarction: new evidence from Grampian region early anistreplase trial (GREAT). $B M \Im$ 1996;312:212-16.

3 Cannon CP, Lambrew CT, Tiefenbrunn AJ, French J, Gore $\mathrm{JM}$, Weaver D, et al. Influence of door-to-balloon time on mortality in primary angioplasty results in 3,648 patients in the Second National Registry of Myocardial Infarction (NRMI-2). F Am Coll Cardiol 1996;27:2:61A

4 Stone GW, Grines CL, Browne KF, Marco J, Rothbaum D, O'Keefe J, et al. Predictors of in-hospital and 6-month outcome after acute myocardial infarction in the repenth outcome after acute myocardial infarction in the reperfusion (PAMI) Trial. $\mathcal{F}$ Am Coll Cardiol 1995;25:370-7.

5 Hochman JS, Boland J, Sleeper LA, Porway M, Brinker J, Col $\mathrm{J}$, et al. Current spectrum of cardiogenic shock and effect of $\mathrm{J}$, et al. Current spectrum of cardiogenic shock and effect of early revascularization on mortality: result
tional registry. Circulation 1995;91:873-81.

6 Cragg DR, Friedmanm HZ, Bonema JD, Jaiyesima I, Ramos RG, Timmis GC, et al. Outcome of patients with myocarRG, Timmis GC, et al. Outcome of patients with myocar-
dial infarction who are ineligible for thrombolytic therapy. dial infarction who are ineligible

7 Brodie BR, Weintraub RA, Stuckey TD, Le Bauer EJ, Katz JD, Kelly TA, et al. Outcomes of direct coronary angioplasty for acute myocardial infarction in candidates and non-candidates for thrombolytic therapy. Am $\mathcal{f}$ Cardiol 1991;67:7-12. 